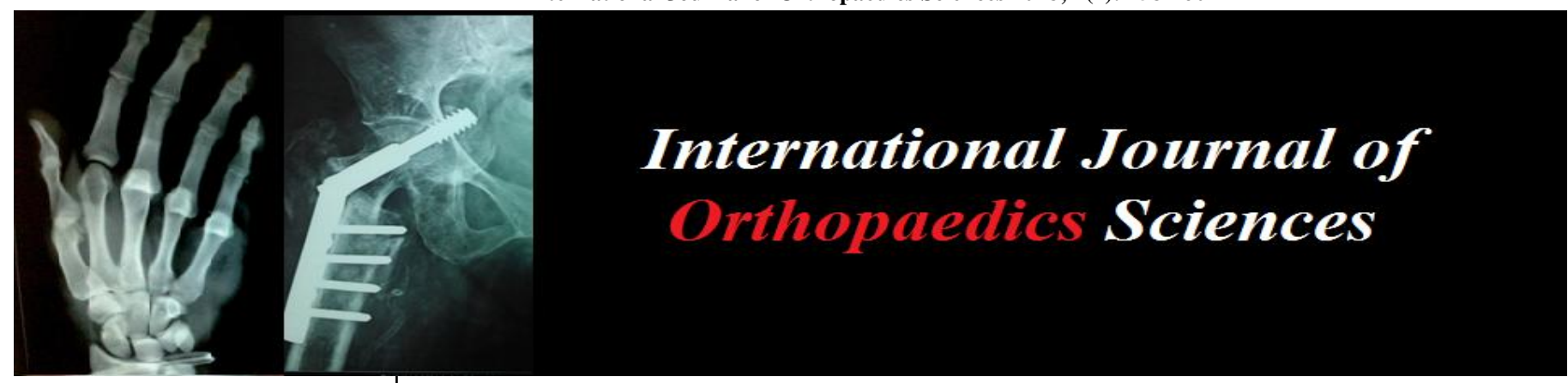

ISSN: $2395-1958$

IJOS 2018; 4(2): 158-160

(C) 2018 IJOS

www.orthopaper.com

Received: 10-02-2018

Accepted: 12-03-2018

RCS Khandelwal

Professor and Head of Unit,

Dept. of Orthopaedics, Seth G S

Medical College and KEM

hospital, Mumbai, Maharashtra,

India

Shubhanshu Bhaladhare

Resident, Dept. of Orthopaedics, Seth GS Medical College and

KEM hospital, Mumbai,

Maharashtra, India

Umang Sanghvi

Resident, Dept. of Orthopaedics, Seth G S Medical College and K

E M hospital, Mumbai,

Maharashtra, India

Spandan Koshire

Resident, Dept. of Orthopaedics,

Seth GS Medical College and

KEM hospital, Mumbai,

Maharashtra, India

Aditya K Agrawal

Assistant Professor, Dept. of

Orthopaedics, Sumandeep

Vidyapeeth, Vadodara, Gujarat,

India
Correspondence

RCS Khandelwal

Professor and Head of Unit,

Dept. of Orthopaedics, Seth G S

Medical College and KEM

hospital, Mumbai, Maharashtra,

India

\section{Three point contouring of 95 degree DCS plate \& its advantages for fixation of sub trochanteric femur fracture}

\author{
RCS Khandelwal, Shubhanshu Bhaladhare, Umang Sanghvi, Spandan \\ Koshire and Aditya K Agrawal
}

DOI: $\underline{\text { https://doi.org/10.22271/ortho.2018.v4.i2c.24 }}$

\begin{abstract}
Introduction: Subtrochateric region is an area of $5 \mathrm{~cm}$ below the lesser trochanter in femur. One of the methods for fixation of subtrochanteric femur fracture is by using 95 angled DCS (Dynamic Compression Screw) barrel plate. Though it's not the best implant for subtrochanteric internal fixation, in our setting it is one of the good option. To overcome the limitations of $95^{\circ} \mathrm{DCS}$ we suggested three point contouring of $95^{\circ}$ DCS which provides it an anatomical reduction and good results.

Material \& Methods: Study includes 38 patients who are admitted in our hospital with subtrochanteric femur fracture out of which 29 were male and 9 were female. All the patients are treated with $95^{\circ} \mathrm{DCS}$ Barrel plate with three point contouring. Patients are then followed up in our Out Patient department.

Results: Out of 38 patients $37(96.4 \%)$ patient had a union without any complication while one patient had non-union which after bone grafting united.

Conclusion: 3 point contouring of 95 DCS gives good result compared to conventional 95 DCS barrel plate.
\end{abstract}

Keywords: subtrochanteric femur fracture, dynamic compression screw barrel plate, contouring, union

\section{Introduction}

Sub trochanteric region includes the region $5 \mathrm{~cm}$ below the lesser trochanter in femur. Fractures in this region are seen commonly in elderly and those with osteoporotic bone or high velocity injuries. Pathological and biomechanical studies have shown that sub trochanteric region is the most stressed area which concentrates stress on implant and thereby difficult to treat due to complications ${ }^{[1,2]}$. Due to predominance of cortical bone, this region presents a more precarious vascularization than the trans-trochanteric region, which makes the consolidation of the fracture difficult ${ }^{[2]}$. Plate fixation has been considered as a known method for fixation of these fractures. The goal of treatment of any sub trochanteric femur fracture is to restore mobility safely and efficiently while minimizing the risk of medical complications. Various modalities of treatment are available for internal fixation of sub trochanteric femur fracture like angled blade plate, $95^{\circ}$ DCS barrel Plate and more recently proximal ante grade nails ${ }^{[3-7]}$. Different treatment modality has there owns good and bad factors. Out of this ante-grade proximal femoral nail showed promising and good results with a high cost compared to other instrumentation. Also nailing is associated with fat embolism and ARDS which has detrimental effects on patients' overall condition. Thus in our setting we preferably use $95^{\circ}$ DCS. Although it was developed for the treatment of distal femur fracture, $95^{\circ}$ DCS has also been widely used for the fixation of sub trochanteric fractures. However, due to the characteristic biomechanics of the sub trochanteric fractures, several authors reported unsatisfactory results like mal-union, nonunion and implant failure. Complex fractures with deficient medial support are associated with high rates of fixation failure. These conditions are very much technically beneficial for closed and fixation using biological method of fixation. Contouring the plate in three regions which make $95^{\circ}$ DCS anatomically oriented to femur give good anatomical reduction in biological plating. 


\section{Aim \& Objectives}

The aim of our study is to find the results of three point contouring of $95^{\circ}$ DCS plate while fixing sub trochanteric femur fracture. All the patients with sub trochanteric femur fracture admitted in our institutes were recruited for the study after taking proper consent. Patient with compound injuries and with fracture in other bone are excluded from the study.

\section{Materials and Methods}

The plate fixation technique includes open and closed reduction method. In this, proper alignment and fracture reduction is the necessary. We had used $95^{\circ}$ DCS barrel plate for fixation of this type of fracture with some minor changes.

\section{Dynamic Condylar Screw with barrel plate}

DCS consist of two parts (Fig 1). One is Lag screw (Richard screw) which is a partially threaded cannulated cancellous screw with a diameter of $12.5 \mathrm{~mm}$ of threaded portion while $8 \mathrm{~mm}$ for non-threaded portion. Other part is DCS barrel plate. The DCS Plate has a $95^{\circ}$ barrel angle, having an advantage of more proximal entry than the DHS Plate and allowing insertion of two or more screws into the calcar. Its two round proximal plate holes permit insertion of $6.5 \mathrm{~mm}$ cancellous Bone Screws, for stable proximal fixation ${ }^{[7]}$. The side plate accommodates $4.5 \mathrm{~mm}$ cortical bone screw, and comes in different hole lengths.

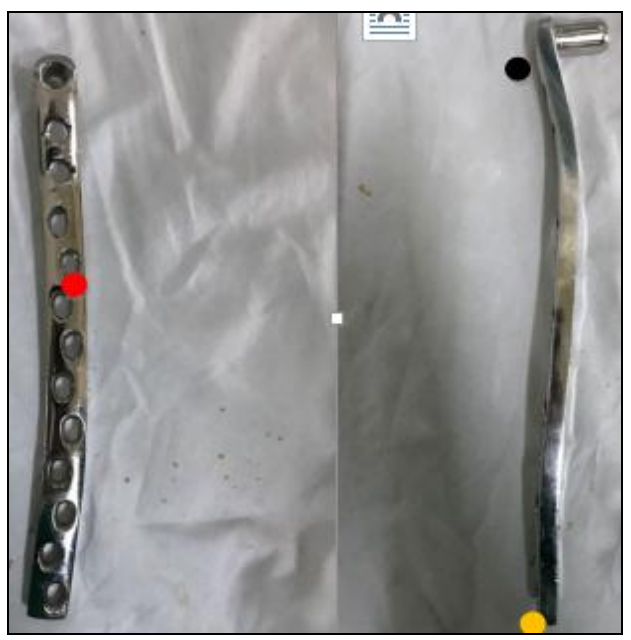

Fig 1: $95^{\circ}$ DCS angle barrel plate with three point contouring

For achieving proper anatomical reduction, we did some minor changes to make the plate more anatomically oriented to the femur. The proposed changes (Fig 1)-

1. Bending the plate in such a way proximally, that the lag screw is inserted in the direction of 110 degrees instead of 95 degrees (Fig1 Black Dot).

2. Bending the plate in sagittal plane up to 5 to 10 degrees, so that it contours according to the anterior bow of the femur(Fig1 Red Dot)

3. Bending the distal one screw in the coronal plane up to 5 to 10 degrees, such that the plate distally is more towards the femur.(Fig1 Yellow Dot)

This bending has to be done with the help of hammer, plate bender and bench press. It is to be done before hand by deciding the size of plate to be used pre operatively, to reduce the operative time by doing the maneuver's intra-operatively. Post-operatively, patient is given 3 days of prophylactic antibiotics and patient is discharged on day 5 and followed up every 15 days till 4 months followed by monthly follow up after 6 months. During follow-ups, routine x-rays are taken.

\section{Radiologic Assessment}

All patients were followed-up at 1, 3, 6, 12 months, and then annually after primary surgery for clinical and radiographic evaluations of the fracture healing process. Radiologic assessments were evaluated using femur AP and lateral images of the affected side and hip AP, lateral, and Lorenz images of the proximal femur. Fracture union was confirmed by two orthopedic surgeons. Union was defined as full painless weight bearing with bridging callus across at least three cortices on AP and lateral views of the femur. (Fig 2)

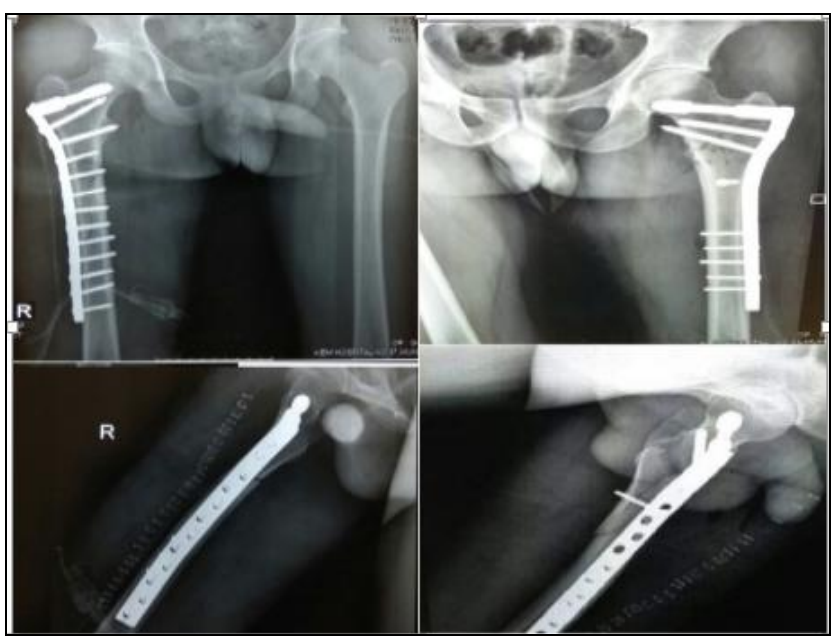

Fig 2: AP and lateral views of the femur showing union

\section{Clinical Assessment}

Operative duration and intraoperative blood loss were recorded. Operative time was defined as the time from skin incision to the end of skin closure. Intraoperative blood loss was analyzed based on the estimated blood loss recorded by the anesthesiologist, which was the approximate amount of blood lost during the surgery. Fat embolism, ARDS, and infection during the follow-up period, and walking ability at the last follow-up visit were assessed using medical records

\section{Results}

The study included 29 males and 9 females. The mean age was 57.7 years (range, 24-85 years). The right femur was involved in 25 patients and the left femur was involved in 13 patients. Types of fracture according to the AO classification were 7 of type $31 \mathrm{~A} 3,22$ of type 32A, 3 of type 32B, and 6 of type $32 \mathrm{C}$. According to the Sheinsheimer classification, there were 3 of type 1,25 of type 2, 2 of type 3, 1 of type 4 , and 7 of type 5. Of these, 18 patients $(47.36 \%)$ had posteromedial defects. The mean surgical timing (time from injury to surgery) was 3.6 days (range, 0-8 days). In the present study, 5 patients received early total care on the day of injury, 20 patients underwent operative treatment 1-5 days after injury, and 13 patients were operated on more than 6 days after injury. The mean follow-up period of the 38 patients in the present study was 22.4 months (range, 12-33 months). The average operative duration was 78.1 minutes (range, 60-120 minutes) and the average intraoperative blood loss was 300 $\mathrm{mL}$ (range, $100-500 \mathrm{~mL}$ ). The mean postoperative coronal alignment of the femur was valgus $1.2^{\circ}$ (range, valgus $6.5^{\circ}$ to varus $9^{\circ}$ ), and the mean shortening of the femur at the last follow-up visit was $0.3 \mathrm{~cm}$ (range, $0-1 \mathrm{~cm}$ ). No patient had a varus or valgus malunion greater than $10^{\circ}$. Fat embolism and ARDS were not observed postoperatively during the hospitalization period of the present study. Union was achieved in 37 patients $(96.4 \%)$ after an average duration of 
5.4 months (range, 3-14 months). One patient develops nonunion after 6months which was bone grafted and had shown union after 6.5 months. There was 1 case of superficial wound infection, and 1 case of distal locking screw breakage during the follow-up period

\section{Discussion}

The purpose of the present study was to determine the advantages of 3 point contouring of 95 degree DCS plate for the fixation of sub trochanteric fractures of the femur. In this study, fracture union was achieved in 37 of 38 patients (96.4\%) without any complications such as fat embolism or ARDS after surgery regardless. Sub trochanteric fractures of the femur constitute $7 \%$ to $34 \%$ of proximal femoral fractures $[1,2]$. This type of fracture typically occurs in active, young individuals due to high energy injury and is often accompanied by multiple injuries, including lung, brain, and abdominal injuries. The predominantly cortical nature of the region, along with the strong muscular attachments and high compressive forces acting medially is responsible for intraoperative difficulties and the high rate of complications associated with the fractures of this region. The incidence of implant failure in patients with sub trochanteric fracture of the femur has been reported as high as $26 \%{ }^{[3-5]}$. Intramedullary nailing has been wildly accepted as a representative surgical option for the treatment of sub trochanteric fracture of the femur. Theoretically, this surgical technique has a number of biomechanical advantages, including a shorter moment arm, greater strength, greater torsional stiffness, and greater loadsharing capacity, compared to plating technique ${ }^{[8,9]}$. In particular, intramedullary nailing is based on the concept of relative biologic fixation, which preserves the vascularity of bone fragment, thereby minimizing soft tissue dissection and blood loss, compared to the traditional open-plating technique $(10,11)$. Nevertheless, intramedullary nailing may provoke fat embolism or additional damage, the so called "second hit" which may cause ARDS postoperatively in patients with multiple traumatic fractures, including lung injury, due to intramedullary reaming during the procedure.

The use of three points contouring of plate for anatomical open reduction or closed reduction was effective, particularly in comminuted sub trochanteric fractures. Based on these results, we hypothesized that plating with the three point contoured plate allows good surgical outcomes in patients with sub trochanteric fracture of the femur. Union has been seen in $96 \%$ of patient in our study which is a significant. Also complications associated with PFN such as fat emboli, implant failure, ARDS, have been minimized. Three points contouring of plate indirectly helps in maintaining the posteromedial cortex of the proximal femur which is one of the important factors in maintaining the reduction and early healing. TPC of 95 DCS can also be used for biological fixation of comminuted sub trochanteric fracture. We in our study used the TPC in biological fixation in about 6 patients with AO type $32 \mathrm{C}$ which had shown early healing and good fracture alignment. Thus the findings of the study demonstrate that fixation using a three point contoured 95 degree DCS plate has a high success rate of fracture union with a low rate of radiologic complications and a low risk of pulmonary complications postoperatively regardless of injury severity, surgical timing, and presence of lung injury.

\section{Conclusion}

Fixation using three point contoured 95 degree DCS plates may represent a good option in patients with sub trochanteric fracture regardless of injury severity, surgical timing, and fracture type. It also has advantage over other implants in regard of ease of surgery. However, a comparative study with other types of fixation is still required.

\section{References}

1. Tordis TG. Stress analysis of the femur. J Biomech. 1969; 2:163-74.

2. Fielding JW, Cochran GV, Zickel RE. Biomechanical characteristics and surgical management of subtrochanteric fractures. Orthop Clin North Am. 1974; 5:629-650.

3. Waddell JP. Subtrochanteric fractures of the femur: a review of 130 patients. J Trauma. 1979; 19:582-592.

4. Velasco RU, Comfort T. Analysis of treatment problems in subtrochanteric fractures of the femur. J Trauma. 1978; 18:513-22.

5. Parker MJ, Dutta BK, Sivaji C, Pryor GA. Sub trochanteric fractures of the femur. Injury. 1997; 28:9195.

6. Bedi A, Le TT. Subtrochanteric femur fractures. Orthopedic Clinics of North America. 2004; 35(4):47383.

7. Sanders R, Regazzoni P. Treatment of sub trochanteric femur fractures using the dynamic condylar screw. Journal of orthopaedic trauma. 1989; 3(3):206-13.

8. Zickel RE. An intramedullary fixation device for the proximal part of the femur. Nine years' experience. J Bone Joint Surg Am. 1976; 58:866-872.

9. Johnson KD, Tencer AF, Sherman MC. Biomechanical factors affecting fracture stability and femoral bursting in closed intramedullary nailing of femoral shaft fractures, with illustrative case presentations. J Orthop Trauma. 1987; 1:1-11.

10. Kraemer WJ, Hearn TC, Powell JN, Mahomed N. Fixation of segmental sub trochanteric fractures. A biomechanical study. Clin Orthop Relat Res. 1996; (332):71-79.

11. Brumback RJ, Toal TR, Jr, Murphy-Zane MS, Novak VP, Belkoff SM. Immediate weight-bearing after treatment of a comminuted fracture of the femoral shaft with a statically locked intramedullary nail. J Bone Joint Surg Am. 1999; 81:1538-1544.

12. Yoon RS, Donegan DJ, Liporace FA. Reducing sub trochanteric femur fractures: tips and tricks, do's and don'ts. J Orthop Trauma. 2015; 29(4):28-33.

13. Celebi L, Can M, Muratli HH, Yagmurlu MF, Yuksel $\mathrm{HY}$, Bicimoğlu A. Indirect reduction and biological internal fixation of comminuted sub trochanteric fractures of the femur. Injury. 2006; 37:740-750. 